\title{
Integración de las TIC en la educación escolar: importancia de la coordinación, la formación y la organización interna de los centros educativos desde un análisis bibliométrico
}

\section{Integration of ICT in school education: importance of coordination, training and internal organization of schools from a bibliometric analysis}

\author{
Juan José Sosa Alonso ${ }^{1}$ \\ https://orcid.org/0000-0001-5615-5536 \\ Anabel Bethencourt Aguilar ${ }^{2}$ \\ https://orcid.org/0000-0002-3823-0835 \\ Universidad de La Laguna, Tenerife, España
}

Recibido: 05-05-2019

Aceptado: 30-07-2019

\section{Cita Recomendada}

Sosa, J. \& Bethencourt, A. (2019). Integración de las TIC en la educación escolar: Importancia de la coordinación, la formación y la organización interna de los centros educativos desde un análisis bibliométrico. Hamut'ay, 6(2), 24-41.

http://dx.doi.org/10.21503/hamu.v6i2.1772

\section{RESUMEN}

El presente artículo es parte de un estudio bibliométrico más amplio, centrado en publicaciones de impacto indexadas a partir del año 2006, referidas al proceso de integración de las tecnologías en el aula. Se presentan los resultados obtenidos de la revisión teórica del tópico de coordinación, organización y asesoramiento dentro de los centros escolares en conjunción con el tópico referido a la formación del profesorado en niveles iniciales, permanente y sus competencias. Los objetivos del estudio son, por un lado, revisar y clasificar la producción académica sobre los tópicos mencionados y, por otro lado, analizar de una muestra seleccionada de trabajos, las principales tendencias de investigación y de contenido. La metodología de investigación utilizada es, por lo tanto, una revisión teórica descriptiva e interpretativa en la que se han revisado un total de 45 artículos de investigación actuales de impacto. Entre las conclusiones, podemos destacar el rol del coordinador o coordinadora TIC como agente clave para el proceso de integración de las tecnologías en la práctica diaria, la necesidad de coordinación y comunicación interna positiva en los centros escolares para que haya un cambio metodológico apoyado en diversos recursos -entre los que cabe mencionar los dispositivos digitales- y un conocimiento integral disciplinar, pedagógico y técnico del profesorado para el buen uso de la tecnología en educación.

Palabras Clave: Escuela, Formación del profesorado, Coordinación centros, Tecnología Educativa, Asesoramiento TIC.

\footnotetext{
${ }^{1}$ Profesor Ayudante-Doctor del Departamento de Didáctica e Investigación Educativa de la Universidad de La Laguna. Licenciado en Pedagogía, licenciado en Psicología y Doctor en Psicología por la ULL. Miembro del grupo de investigación EDULLAB (Laboratorio de Educación y Nuevas Tecnologías de la ULL) y miembro del grupo de investigación POSASUE (Poder, Saber y Subjetividad en Educación). E-mail: jsosalo@ull.edu.es

${ }^{2}$ Doctoranda en el Programa de Educación en la Universidad de La Laguna. Máster en Educación Digital por UNEX y Graduada en Pedagogía por la ULL. Miembro del grupo de investigación EDULLAB (Laboratorio de Educación y Nuevas Tecnologías de la ULL). E-mail:abethenc@ull.edu.es
} 


\begin{abstract}
This article is part of a broader bibliometric study, focused on impact publications indexed since 2006, referring to the process of integration of technologies in the classroom. The results obtained from the theoretical review of the topic of coordination, organization, and counseling within the school centers are presented in conjunction with the topic referring to teacher training at initial, permanent levels and their competences. The objectives of the study are to review and classify the academic production on the mentioned topics on one side and to analyze the main research and content trends of a selected sample of works on the other. The research methodology used is, therefore, a descriptive and interpretive theoretical review in which a total of 45 current impact research articles have been reviewed. Among the conclusions, we can highlight the role of the coordinator or ICT coordinator as a key agent for the process of integrating technologies into daily practice, the need for coordination and positive internal communication in schools so that there is a methodological change supported by diverse resources - among which digital devices should be mentioned - and a comprehensive disciplinary, pedagogical and technical knowledge of teachers for the proper use of technology in education.
\end{abstract}

Keywords: School, Teacher Training, Coordination centers, Educational Technology, ICT Consulting.

\section{INTRODUCCIÓN}

El estudio de las tecnologías en educación es una de las temáticas que mayor auge ha tenido durante la última década. Un ejemplo de ello es que, actualmente, la revista mejor posicionada a nivel español -según Google Metrics-, es la revista Comunicar dedicada a publicar artículos de investigación sobre el ámbito de la comunicación, tecnologías y educación. Entre muchas de sus temáticas, se encuentran el uso de las redes sociales en educación (Tuzel \& Hobbs, 2017; Hershkovizt \& Forkosh-Baruch, 2017; Tur, Marín-Juarros, \& Carpenter, 2017), las redes de aprendizaje colaborativas y experienciales para la formación (Ramírez-Montoya \& García-Peñalvo, 2018; Díez-Gutiérrez \& Díaz-Nafría, 2018; Álvarez-Arregui et al., 2017; Velandia-Mesa, Serrano-Pastor, \& Martínez-Segura 2017), el estudio sobre las metodologías innovadoras para la mejora en la educación (Muñoz-Repiso, Gómez-Pablos, \& García 2014; Pérez, Fernández, \& Braojos 2010), análisis sobre la alfabetización digital, mediática y para los medios (Pérez-Rodríguez \& Ponce, 2012; Martín \& Tyner, 2012), entre muchos otros. Se sabe que, desde el punto de vista bibliométrico, la temáti- ca de las tecnologías en educación tiene un gran impacto y presencia en la literatura académica. La amplia cantidad de artículos de investigación especializados en esta temática manifiesta la necesidad de realizar una presentación ordenada y sintética de la producción, lo cual conlleva a la necesidad de conocer y revisar los resultados obtenidos para extraer líneas comunes.

Este artículo de investigación se centra exclusivamente en el análisis de dos tópicos concretos dentro de la compleja temática de las TIC en educación. El trabajo nace con la finalidad de indagar sobre la presencia de recursos bibliográficos orientados a exaltar la relevancia de la coordinación, el asesoramiento y la organización para la buena implementación de las tecnologías en el centro; así como la importancia que le da la literatura académica a la mejora competencial y formativa del profesorado en los aspectos técnicos-didácticos del uso de las tecnologías en educación. Estos dos tópicos comentados constan de gran importancia en el uso didáctico de las tecnologías en educación.

En definitiva, el propósito de este trabajo es realizar una presentación sintética y ordenada de las 
principales conclusiones de una selección representativa y relevante de trabajos de investigación sobre el fenómeno de las TIC en el centro escolar y en el profesorado. De este modo, los objetivos son: i. Revisar y clasificar la producción sobre la coordinación, el asesoramiento y la organización de centro, así como la formación del profesorado en la implementación de las tecnologías en la educación. ii. Analizar el contenido y extraer conclusiones sobre las principales barreras y su nivel de relevancia explicitada en las investigaciones educativas de impacto.

El primer objetivo está más relacionado con el análisis bibliométrico pertinente a la temática (número de publicaciones, tipología de publicación, líneas de investigación más frecuentes, metodologías e instrumentos de investigación más usuales); mientras que el segundo objetivo responde también a un análisis de contenido sobre la temática ya explicitada.

\section{Materiales Y Métodos}

\section{Participantes}

La muestra seleccionada estuvo conformada por una revisión de materiales bibliográficos, por ser este un análisis de la literatura existente sobre la "Formación inicial, permanente y en competencias" y "Coordinación, asesoramiento y organización de centro". Los criterios de inclusión que se consideraron para realizar la revisión teórica y bibliométrica se describen en la tabla 1 .

\section{Tabla 1}

Criterios de inclusión para la revisión teórica y bibliométrica

\begin{tabular}{|c|c|c|c|c|}
\hline $\begin{array}{l}\text { Tipo de } \\
\text { material }\end{array}$ & Idioma & $\begin{array}{l}\text { Inter- } \\
\text { valo de } \\
\text { fechas }\end{array}$ & $\begin{array}{l}\text { Tipo de } \\
\text { revistas }\end{array}$ & $\begin{array}{c}\text { Base de } \\
\text { datos }\end{array}$ \\
\hline $\begin{array}{l}\text { Artículos de } \\
\text { investigación, } \\
\text { libros en for- } \\
\text { mato digital } \\
\text { físico, tesis } \\
\text { doctorales, } \\
\text { etc. }\end{array}$ & Español & $\begin{array}{l}5 \text { años } \\
\text { Inicia } \\
2006 \\
\text { al } \\
2019\end{array}$ & $\begin{array}{l}\text { Revistas } \\
\text { españolas } \\
\text { especiali- } \\
\text { zadas }\end{array}$ & $\begin{array}{l}\text { Web of } \\
\text { Science, } \\
\text { Scopus, } \\
\text { Miar o } \\
\text { Google } \\
\text { Scholar } \\
\text { Metrics }\end{array}$ \\
\hline
\end{tabular}

Fuente: Elaboración propia (2019).

Los aspectos descritos en la tabla 1 posibilitarán extraer conclusiones sobre el auge de esta temática en concreto y sobre su nivel de importancia para las investigaciones, ya que los artículos seleccionados forman parte de las revistas indexadas con indicadores evaluativos de calidad con un índice $\mathrm{H}$ relevante. De este modo, la muestra bibliográfica seleccionada esta alojada en la plataforma de Punto Q de la Universidad de La Laguna de las 32 revistas de impacto académicas españolas y/o iberoamericanas, en la tabla 2 se detalla el listado de las mismas.

Tabla 2

Listado de revistas seleccionadas para la muestra

\begin{tabular}{|c|c|c|}
\hline \multicolumn{3}{|c|}{ Revistas seleccionadas de la muestra } \\
\hline $\begin{array}{l}\text { - @tic } \\
\text { - Bordón } \\
\text { - Comunicar } \\
\text { - Cultura y Edu- } \\
\text { cación } \\
\text { - Digital Education } \\
\text { Review } \\
\text { - Educación XXI } \\
\text { - Elec. Journal } \\
\text { of Research } \\
\text { in Educational } \\
\text { Psychology } \\
\text { - Enseñanza de } \\
\text { las ciencias } \\
\text { - Estudios sobre } \\
\text { Educación (ESE) } \\
\text { - Infancia y Apren- } \\
\text { dizaje } \\
\text { - Intangible Capital } \\
\text { - Pixel-Bit } \\
\text { - Profesorado } \\
\text { - Revista lbe- } \\
\text { roamericana de } \\
\text { Educación }\end{array}$ & $\begin{array}{l}\text { - Revista Com- } \\
\text { plutense de } \\
\text { Educación } \\
\text { - Revista Electróni- } \\
\text { ca Interuniversita- } \\
\text { ria de Formación } \\
\text { del Profesorado } \\
\text { - Revista Elec- } \\
\text { trónica Tecno- } \\
\text { logía Educativa } \\
\text { EDUTEC } \\
\text { - Revista de Do- } \\
\text { cencia Universita- } \\
\text { ria (REDU) } \\
\text { - Revista de Edu- } \\
\text { cación, Revista } \\
\text { de Educación a } \\
\text { Distancia (RED) } \\
\text { - Revista de } \\
\text { Investigación } \\
\text { Educativa (RIE) } \\
\text { - Revista de Psico- } \\
\text { didáctica } \\
\text { - Revista Electróni- } \\
\text { ca de Investiga- } \\
\text { ción y Evaluación } \\
\text { Educativa } \\
\text { (RELIEVE) }\end{array}$ & $\begin{array}{l}\text { - Revista } \\
\text { Electrónica de } \\
\text { Investigación } \\
\text { Educativa } \\
\text { - Revista } \\
\text { Española de } \\
\text { Orientación y } \\
\text { Psicopedago- } \\
\text { gía (REOP) } \\
\text { - Revista } \\
\text { Española de } \\
\text { Pedagogía } \\
\text { - Revista Ibe- } \\
\text { roamericana de } \\
\text { Educación, } \\
\text { - Revista } \\
\text { Iberoamericana } \\
\text { de Educación } \\
\text { a Distancia } \\
\text { (RIED) } \\
\text { - RUSC: Revista } \\
\text { de Universidad } \\
\text { y Sociedad del } \\
\text { Conocimiento } \\
\text { - Teoría de la } \\
\text { Educación } \\
\text { - Educación y } \\
\text { Cultura en la } \\
\text { Sociedad de la } \\
\text { Información }\end{array}$ \\
\hline
\end{tabular}

Fuente: Elaboración propia (2019).

Como resultado del proceso de revisión, con relación a los dos tópicos de este estudio, se tuvo como muestra final, para la fase de análisis de contenido, los 48 artículos de investigación que se detallan en los anexos. 


\section{Tipo y diseño de estudio}

El tipo de estudio es una revisión teórica interpretativa (Fernández-Ríos \& Buela-Casal (2009), los cuales brindan contribuciones muy importantes en el campo de la ciencia, dado que pueden aportar un excelente resumen del estado actual de la investigación sobre un tema, y de diseño descriptivo (Guirao-Goris, Olmedo, \& Ferrer, 2008) apoyado en datos de tipo cuantitativos y cualitativos, a través de la revisión bibliográfica que selecciona la información relevante perteneciente a una temática concreta y la presenta después de ser clasificada y revisada, lo que ayudará a sacar conclusiones sobre los tópicos seleccionados. Este estudio surge de un análisis bibliométrico de las tecnologías educativas y el sistema escolar en diferentes categorías, pero sólo se presentan los datos obtenidos de formación inicial, permanente y en competencias TIC del profesorado y del nivel de coordinación, asesoramiento y organización de los centros educativos para el proceso de implementación de las tecnologías a la práctica diaria.

De este modo, el estudio refleja la situación, para el periodo contemplado, a través de un análisis de la producción amplia de acuerdo con los tipos de recursos (artículos de investigación, libros en formato digital físico, tesis doctorales, etc.), y ordenada en intervalos de años (cada 5 años hasta la actualidad). Por último, se presenta un análisis de carácter bibliométrico (descriptores más habituales, metodologías de investigación y técnicas e instrumentos más usuales) y teórico (análisis de contenido).

\section{Procedimiento y análisis de la información}

El presente artículo de investigación forma parte de un estudio más amplio donde se ha realizado una categorización de las TIC en el sistema educativo escolar. Los tópicos utilizados fueron aquellos más usuales de la literatura académica en TIC como "Política Educativa y tendencia TIC", "Formación inicial, permanente y en competencias", "Coordinación, asesoramiento y organización de centro", "Metodología, usos y recursos TIC", "Impacto en el aprendizaje con TIC" y "Percepción, opinión y expectativas".
Primera etapa (análisis cuantitativo bibliométrico): Se realiza una revisión de la literatura en el metabuscador "Punto Q", gestionado por el Servicio de Biblioteca de la Universidad de La Laguna, y en diferentes revistas, obteniéndose un listado de recursos bibliográfico ordenado gracias a software de manejo de bibliografía Refword Plus y Zotero. Posteriormente a la búsqueda sistemática por palabras claves, se depuraron los listados obtenidos o eliminaron las duplicidades y aquellos recursos que no se relacionan con la temática concreta del estudio. Por último, como se ha comentado, se hizo una clasificación conforme al tipo de recurso y al año de las publicaciones, para extraer el estado del arte bibliográfico sobre estos tópicos concretos.

Segunda etapa (análisis cualitativo de contenido, sobre una muestra seleccionada de artículos representativos): En esta segunda etapa, se elabora un ranking de revistas teniendo en consideración los indicadores de calidad comunes en la literatura académica, de aplicación en entornos y plataformas como Web of Science, Scopus, Fecyt, Erih, Circ, Carhus, Miar, Google Scholar Metrics, para seleccionar la muestra de artículos de investigación del que se procederá a realizar el análisis. La clasificación de estos artículos se hizo atendiendo a los principales descriptores dentro de los tópicos categorizados, la metodología de investigación (siguiendo la clasificación introducida en su momento por Arnal, del Rincón, \& Latorre, 1992) y atendiendo a los instrumentos de análisis de la información. A partir de estos datos iniciales de la muestra seleccionada, se procede a realizar el análisis de contenido pormenorizado, con apoyo en ATLAS.ti, para cada artículo de investigación. Este procedimiento posibilita la extracción de conclusiones en torno a cómo se realizan las investigaciones y qué datos se difunden sobre formación y competencias digitales en el profesorado y la coordinación, asesoramiento y organización de los centros TIC.

\section{Resultados}

A continuación, se describen los resultados obtenidos de analizar la mejora competencial y forma- 
tiva del profesorado en los aspectos técnicos-didácticos del uso de las tecnologías en educación.

Formación inicial, permanente y en competencias del profesorado

Comenzando por el análisis bibliométrico (cuantitativo) del tópico de "Formación inicial, permanente y en competencias" se ha buscado en las bases de datos anteriormente comentadas, obteniéndose los siguientes resultados en español y a partir del año 1995 hasta la actualidad.

\section{Tabla 3}

Relación del tópico "Formación inicial, permanente y en competencias" con la producción obtenida

\begin{tabular}{lc}
\hline Sub-tòpico & $\begin{array}{c}\text { Número } \\
\text { de refe- } \\
\text { rencias }\end{array}$ \\
\hline Formación del profesorado en TIC & 913 \\
Formación y competencia del profesorado en TIC & 330 \\
Formación digital del profesorado & 1285 \\
Formación digital del profesorado en TIC & 294 \\
\hline
\end{tabular}

Fuente: Elaboración propia (2019) .

Posteriormente, se criban aquellos recursos que no están relacionados con la temática en concreto. El tipo de recurso que más producción tiene sobre la formación del profesorado en la tecnología y educación es el artículo de investigación -total de 410-. Los recursos como libros, tesis doctorales, etc., tienen un total de 84 recursos distintos. Esto hace que los artículos de investigación superen con creces al total restante.

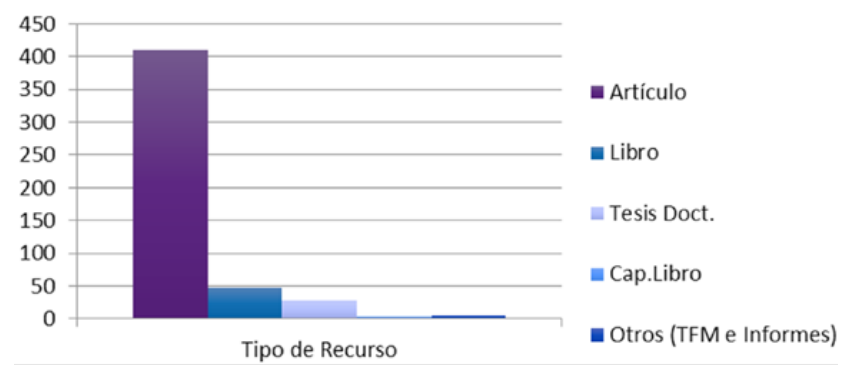

Figura 1.

Tópico de Formación del profesorado por tipo de recurso.

Por otro lado, es el intervalo de años de 2010 a 2014 la que más producción tiene -178 en total-.
No obstante, es llamativo que, en comparación con el intervalo más actual, sólo se distinguen por 43 recursos. Teniendo en cuenta que todavía estamos a mitad de año, puede que rivalice con el intervalo de tiempo anterior, aunque puede ser menos posible que termine superándolo. La preocupación sobre la formación del profesorado ha hecho que se desarrolle cada vez más investigaciones y ensayos orientados a debatir sobre las competencias y capacidades que los agentes educativos disponen o deberían disponer.

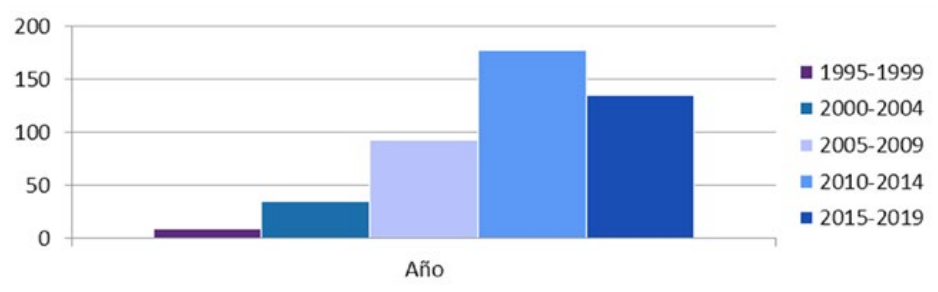

Figura 2.

Tópico de Formación del profesorado por intervalo de año de producción.

Por otro lado, desde una perspectiva más cualitativa, el análisis de contenido para este tópico se ha desarrollado sobre una muestra concreta de 25 artículos de investigación, que han sido escogidos por su representatividad e impacto.

Como hemos comentado en el apartado de procedimiento metodológico, se han clasificado conforme el descriptor, la metodología y los instrumentos utilizados. En cuanto a las subtemáticas, de los 25 artículos encontramos que hay mayor predisposición para investigar las competencias TIC del profesorado - 14 artículos en total de la muestra seleccionada-, que la formación permanente, inicial u otras tendencias. Estas investigaciones analizadas se caracterizan por tener una perspectiva metodológica empírico-analítica -18 artículos de investigación en total- utilizando el cuestionario como recogida de información frente al total de otros instrumentos como las entrevistas, el análisis de contenido, o la realización de grupos de discusión, entre otros.

La mayoría de los artículos analizados consideran al profesorado como uno de los agentes educativos claves para la adecuada integración de las tecnologías en la práctica diaria (Gewerc \& Montero, 2013; Prendes, Castañeda, \& Gutiérrez, 
2010). Precisamente por ello, investigaciones consultadas apuestan por analizar la formación del profesorado antes de que desarrolle su servicio, y posteriormente, una vez que ya tiene un recorrido como profesional educativo dentro del sistema escolar.
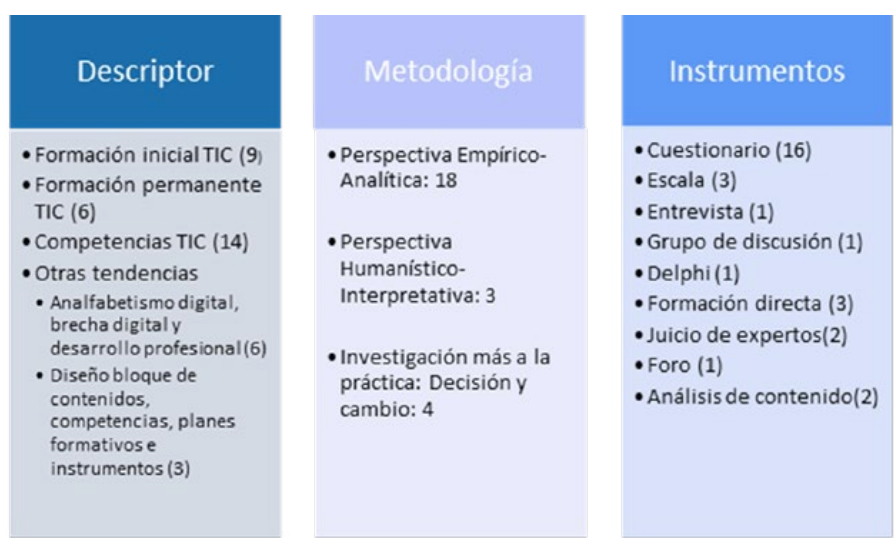

Figura 3.

Descriptores, metodologías e instrumentos utilizados en la producción académica.

En los intervalos anteriores al año 2016, era más frecuente que las investigaciones apuntasen a la necesidad de formación del profesorado en los aspectos técnicos y didácticos para compensar, de algún modo, con las capacidades digitales entendidas como "innatas" del alumnado. Se pensaba que, por el simple hecho de haber nacido en una etapa donde la cultura digital está mucho más extendida, el alumnado tenía unas habilidades hasta ahora desconocidas por el profesorado. Esta argumentación se extendía con el desencuentro de las generaciones entre el profesorado y quienes han nacido en la generación $Z$, generación $Y$, entre otras. La creencia de que, por el simple hecho de haber nacido en una era donde las tecnologías están mucho más presentes, hacía cuestionar la habilidad del profesorado -inmigrantes digitalesen comparación con las habilidades del alumnado -generación interactiva o nativo digital-, nos hace reflexionar que la cercanía a los dispositivos tecnológicos no garantiza un uso adecuado (Gómez, Cañas, Gutiérrez \& Martín-Díaz, 2014).

Esta creencia generalizada ha perdido fuerza, en la medida en la que surgen interpretaciones que apuntan a que, aunque el alumnado esté más familiarizado con las tecnologías no lo hace más competente, y mucho menos que las utilice de forma adecuada. Las investigaciones manifiestan la importante función del profesorado para que el alumnado desarrolle la competencia tecnológica y se aproveche de las posibilidades educativas en beneficio de su aprendizaje. Bajo este planteamiento, el profesorado es el responsable que debe estar lo suficientemente formado para mejorar la competencia de su alumnado (Fernández \& Fernández, 2016; Gómez, Cañas, Gutiérrez, \& Martín, 2014; Cortina-Pérez, Gallardo-Vigil, Jiménez-Jiménez, \& Trujillo-Torres, 2014). Siguiendo el hilo de este planteamiento, se encontró investigaciones que apuntan en el mismo sentido generacional, advirtiendo que no es el profesorado más joven el que utiliza las tecnologías de forma más asidua, sino aquel que ya tiene cierto bagaje, experiencias y destrezas (Area, Hernández, \& Sosa, 2016).

Ante esta situación, las competencias del profesorado se tornan elementos cruciales para transformar la práctica educativa, aunque hay quienes definen la competencia digital de forma diferente. Un ejemplo sobre el esfuerzo en clarificar qué supone las competencias dígales del profesorado es el Marco Común de Competencia Digital Docente, donde se considera las siguientes cinco líneas competenciales: Información y alfabetización informacional, Comunicación y colaboración, Creación de contenidos digitales, Seguridad y la Resolución de problemas. Por otro lado, según algunos artículos de investigación (Roig, Mengual-Andrés \& Quinto-Medrano, 2015), el profesorado ha de tener un cúmulo de competencias más allá del mero uso digital para desarrollar buenas prácticas con tecnología en el aula. Estas habilidades están relacionadas con la triangulación de conocimientos disciplinares, tecnológicos y pedagógicos (Prendes, Castañeda, \& Gutiérrez, 2010; Cabero, 2014; Fernández, Fernández, \& Cebreiro, 2016). Sin entrar en el debate de cuál de ellas es más relevante, el profesorado debe mejorar su conocimiento sobre la funcionalidad de las herramientas digitales, sobre su uso en su asignatura, así como en las estrategias metodológicas y educativas para concebir las posibilidades reales de éstas en el aula (Fernández \& Fernández, 2016; Cózar, Moya, Hernández \& Hernández, 2015; García et al., 2014; Cerveró, Rodríguez, Meliá, \& Alonso, 2011). La comunidad educa- 
tiva debe superar el rechazo a las tecnologías de forma absoluta, así como la fascinación superficial por el sólo hecho de ser digital. De este modo, se empezarían a utilizar de un modo más asiduo, útil, educativo y coherente, más que para prácticas concretas de planificación de las clases u otras tareas secundarias de organización.

En las investigaciones consultadas, se encuentra que la formación digital del profesorado tiende a ser baja. Desde la formación inicial, las experiencias educativas que ha vivenciado el alumnado egresado suelen repetirse una vez que éste forme parte del equipo profesional. Si no ha vivenciado nunca la utilización de la tecnología como un recurso ajustado y naturalizado en la práctica diaria, es más complejo que vea las posibilidades didácticas reales que tienen estos recursos. En este sentido es necesario que se desarrollen prácticas innovadoras en la Universidad para que el profesorado futuro disponga de múltiples ejemplos donde se utilicen los recursos analógicos y digitales sin priorizar exclusivamente en el libro de texto. Además de esto, el futuro docente debe tener conocimiento sobre las TIC y el apoyo educativo al alumnado con Necesidades Específicas de Apoyo Educativo (NEAE), ya que algunas investigaciones también demuestran la nula formación en torno a esta temática, de vital importancia para la inclusión educativa de capacidades diversas (Llorente \& Toledo, 2016; Tello \& Cascales, 2015).

Con respecto a la formación permanente, los resultados sobre su formación y competencia parecen también ser bajos. El profesorado siente que su nivel de formación es mucho menor de lo que en realidad podría serlo, sin embargo, se deben seguir realizando esfuerzos que mejoren la formación de los agentes educativos además de mejorar su seguridad para llevar a cabo prácticas innovadoras con tecnología.

Actualmente, parece que el profesorado en formación permanente tiende a aprender de manera autodidacta (Prendes, Castañeda, \& Gutiérrez, 2010; Boza \& Conde, 2015; Cerveró, Rodríguez, Meliá, \& Alonso, 2011; Colás-Bravo \& Hernández Portero, 2014; Llorente \& Toledo, 2016). Esto puede deberse a varios motivos: por un lado, la escasez de tiempo del profesorado conlleva a que, si quiere seguir formándose debe organizarse y buscar recursos para mejorar como profesional; y, por otro lado, el profesorado considera que no hay recetas ampliamente extendidas que ayuden a la integración de las tecnologías, sino que, más bien es el conocimiento sobre su propia disciplina, su propia aula o los intereses y gustos de su alumnado, lo que posibilita el desarrollo de buenas prácticas con TIC.

Si éste acude a cursos presenciales o se matricula en cursos online para su formación, debe traducir todo el conocimiento en abstracto a sus propias capacidades y circunstancias, complejizando el impacto hacia la mejora. La formación del profesorado sería más ajustada si pudiera aprender durante su propio contexto real, debido a que los esfuerzos por mejorar las competencias TIC no siempre se vinculan con las vivencias cotidianas y problemáticas que surgen en el propio seno de su centro o en su aula.

En este siguiente apartado, se describen los resultados obtenidos relacionados al objetivo presencia de recursos bibliográficos orientados a exaltar la relevancia de la coordinación, el asesoramiento y la organización para la buena implementación de las tecnologías en el centro.

\section{Coordinación, asesoramiento y organización del centro}

La coordinación, asesoramiento y la organización del centro para la integración de las tecnologías es un fundamento esencial. En este tópico, se han buscado en las bases de datos ya señaladas, obteniéndose los siguientes resultados:

\section{Tabla 4}

Relación del tópico "Coordinación, asesoramiento y organización del centro” con la producción obtenida

\begin{tabular}{lc}
\hline Sub-tòpico & $\begin{array}{c}\text { Número } \\
\text { de refe- } \\
\text { rencias }\end{array}$ \\
\hline Coordinación TIC & 259 \\
Asesoramiento TIC & 86 \\
Coordinación educativa en TIC & 87 \\
Centros TIC & 1136 \\
\hline
\end{tabular}

Fuente: Elaboración propia (2019). 
Posteriormente, siguiendo el procedimiento ya comentado, se limpian las listas obtenidas para eliminar aquellos documentos que no están relacionados con la temática en concreto, que pertenecen a otros niveles que no sea la educación básica, que no esté centrado en las tecnologías en la educación o que no traten de la coordinación, el asesoramiento, la comunicación o la organización del centro para el buen desarrollo de las tecnologías.

Como en el caso anterior, y en la fase de análisis estrictamente cuantitativo, los artículos de investigación son los más frecuentes, con un total de 66 artículos (Figura 4). El dato de los artículos de investigación supera con creces la cantidad total de los otros recursos como libros, tesis, capítulos de libros, etc. Por lo tanto, se puede extraer como conclusión que esta temática más específica se suele trabajar concretamente en el formato de artículos que en otras que exigen mayor detenimiento.

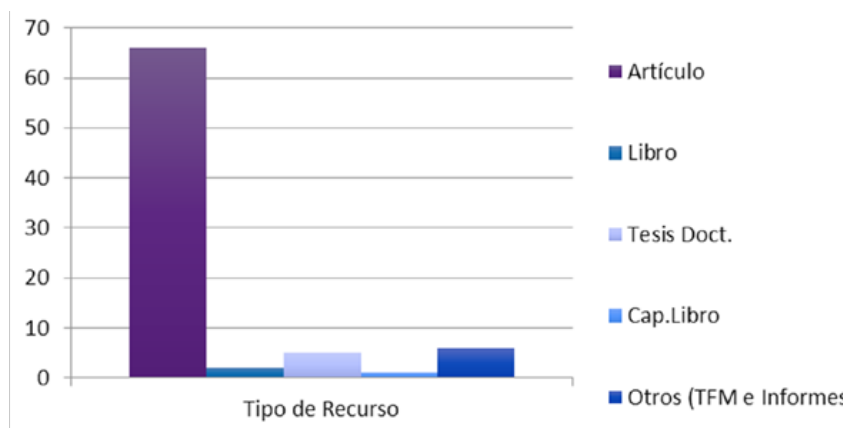

Figura 4.

Tópico de Coordinación y organización por tipo de recurso.

La cantidad obtenida en los intervalos de tiempo anteriores al 2009 suman un total de 22 trabajos académicos. El intervalo de tiempo de 2010-2014 tiene unos 32 recursos; mientras que el intervalo más actual tiene unos 26 en total. Por lo que, desde el 2010 hasta el 2019, aumenta la cifra en unos 36 recursos más sobre esta temática Es conveniente mencionar que el último intervalo de tiempo no ha finalizado, y a lo largo de este año puede haber más recursos destinados a la coordinación de centros como a la organización a los agentes educativos TIC.

En la fase de análisis cualitativa (de contenido) de los artículos de investigación seleccionados -un total de 23 artículos-, se observa que, en su mayo-

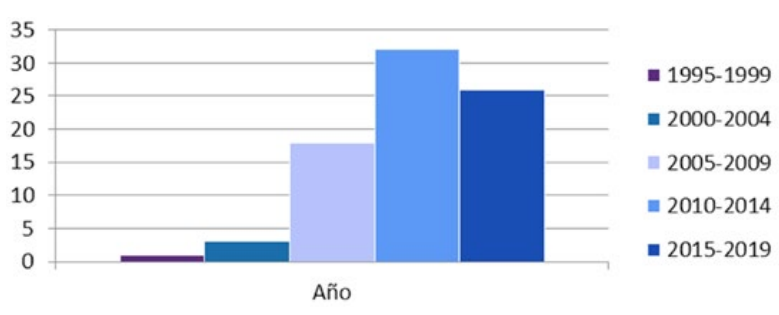

Figura 5.

Tópico de Coordinación y organización por intervalo de año de producción.

ría están más centrados en la organización de centros TIC, en primer lugar y, en la coordinación TIC, en segundo lugar. Con un total de 13 artículos de investigación que hablan exclusivamente de la organización de centros en la utilización de la tecnología, frente al total de 7 artículos para la coordinación. Posteriormente le sigue la investigación dedicada a la dirección de centros y al papel de la familia, cada vez más presente en los artículos de investigación dedicados a analizar las TIC en educación.
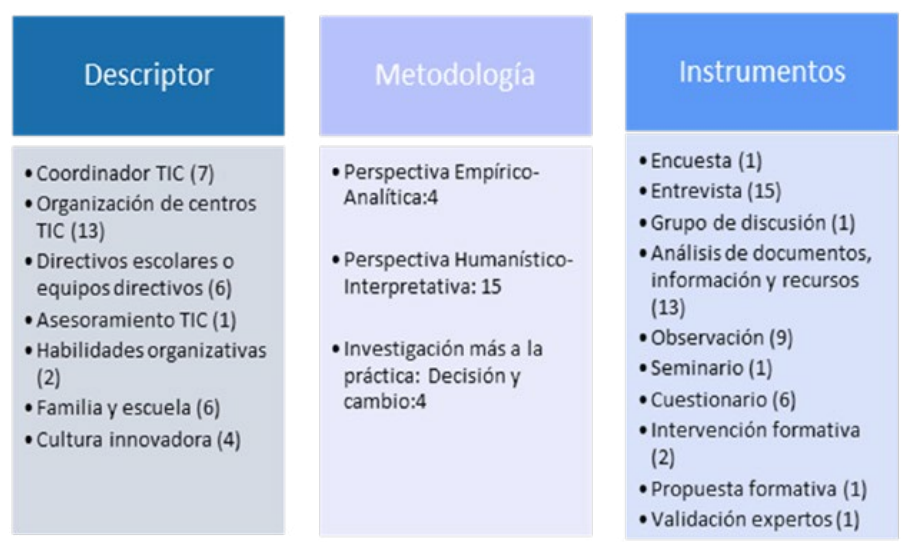

Figura 6.

Descriptores, metodologías e instrumentos utilizados en la producción académica.

Con respecto a la metodología de investigación llevada a cabo por estas investigaciones, tenemos datos que afirman la presencia de una perspectiva más humanística e interpretativa -con un total de 15 artículos bajo esta metodología- que las tendencias empíricas-analíticas o la investigación dedicada a la acción y mejora práctica - con un total entre los 2 de 8 artículos-. En esta ocasión, la presencia de diferentes instrumentos de recogida de la información es notable, encontrándonos con un total de 15 entrevistas, 13 análisis de do- 
cumentos, 9 observaciones, 6 cuestionarios, entre otros. En el caso de los instrumentos como grupos de discusión o validación de expertos, los artículos de investigación explican que han sido seleccionados para dar rigor a los instrumentos y criterios seleccionados, o mayor fiabilidad a los resultados obtenidos.

Los artículos de investigación seleccionados afirman que el coordinador o coordinadora TIC es una pieza clave para la integración de las tecnologías en el sistema educativo (Espuny, Gisbert, Coiduras, \& González, 2012; Hernández, Castro, \& Vega, 2011; Valverde \& Sosa, 2014; Méndez Garido \& Delgado García, 2016; Sosa, Peligros, \& Díaz, 2010). Este agente utiliza en su vida diaria las tecnologías, además de ser una persona que lleva en un mismo centro entre tres a seis años. Esto hace que tenga un conocimiento profundo sobre la organización, dirección, cuerpo de profesionales que trabaja en él y las características de su entorno (Espuny, Gisbert, Coiduras, \& González, 2012; Hernández, Castro, \& Vega, 2011). Además, la persona que asume la coordinación TIC en un centro educativo se le describe por tener una responsabilidad de liderazgo que, de forma autodidacta, asume diversas tareas complejas para planificar, implementar y posibilitar el cambio en la dinámica del aula y centro (Hernández, Castro, \& Vega, 2011).

Una de las principales características que encontramos en torno al papel del coordinador TIC es la ambigüedad de sus funciones (Valverde $\&$ Sosa, 2014; Espuny, Gisbert, Coiduras, \& González, 2012; Hernández, Castro, \& Vega, 2011). Este agente educativo por su conocimiento y tendencia personal estaría preparado para asumir funciones directamente relacionadas con el uso adecuado de la tecnología dentro de la práctica diaria, aunque la realidad en muchos centros educativos es que está limitado a las tareas de manteniendo de recursos y solución de problemas técnicos (Hernández \& Sosa, 2016; Llamas \& Ruiz, 2015; Espuny, Gisbert, Coiduras, \& González, 2012; Sosa, Peligros, \& Díaz, 2010). Estas tareas imposibilitan aquellas otras funciones más coherentes a su perfil educativo como el formar al cuerpo de profesores y profesoras del centro.
En muchas comunidades, el coordinador/a TIC tiene una sobrecarga horaria en su trabajo, además de no estar remunerado por este esfuerzo adicional que realiza; mientras que en otras comunidades tiene beneficios profesionales y se le reconoce su labor (Méndez Garido \& Delgado García, 2016; Espuny, Gisbert, Coiduras, \& González, 2012; Sosa, Peligros, \& Díaz, 2010). Esta ambigüedad complica la labor del coordinador/a, imposibilitando el desarrollo de labores de formación, asesoramiento y cambio dentro de la dinámica del centro, por estar obligado a asumir otras tareas en las que se pierde tiempo y esfuerzo. En este caso, es necesario que las Administraciones educativas se planteen crear equipos técnicos de zona o similar, que posibilite el apoyo técnico cuando haya un problema de esta índole. Las Administraciones educativas deben potenciar más esta figura, clarificando sus funciones, y dotarle de recursos que ayuden a su formación que, a su vez, repercutirá directamente en la del centro.

El asesor especialista dentro de la realidad educativa canaria, también parece ser un agente clave con un conocimiento técnico y pedagógico en tecnologías, además de un fundamental apoyo, formador y facilitador (Hernández \& Sosa, 2016b). En este sentido, el asesor debe conocer las características propias de los centros, con la capacidad suficiente para adaptar sus estrategias individuales, procesuales y de resolución (Hernández \& Sosa, 2016 a y b).

Por otro lado, el equipo directivo debe tener el suficiente liderazgo para responder a los cambios que suponen la utilización de las tecnologías en la educación. Se necesitan, por lo tanto, una organización y liderazgo pedagógico para dotar de los recursos adecuados al coordinador TIC y contribuir a fomentar la implicación del profesorado en el centro (Sosa, Peligros, \& Díaz, 2010; Morales, Lorenzo, \& Trujillo, 2008; Hernández, \& Sosa, 2016a; Jiménez, León, Correa, \& Ibáńez, 2008).

Las investigaciones seleccionadas demuestran que un equipo directivo que tenga una visión y unos principios de integración de las TIC en el centro coherentes aumenta las posibilidades de optimizar el uso de buenas prácticas TIC en el aula. No obstante, se necesita la colaboración del cuerpo pro- 
fesional al completo para que germine y muestre cambios en el proceso de enseñanza y aprendizaje. Una estrategia de liderazgo flexible parece ser más eficiente que un liderazgo autoritario (Valverde \& Sosa, 2014; Sosa, Peligros, \& Díaz, 2010; Aguaded \& Tirado, R, 2010; Jiménez, León, Correa, \& Ibáńez, 2008; Hernández \& Sosa, 2016b). El equipo directivo debe comprender las iniciativas del profesorado para innovar, siendo competentes para proponer opciones si estas no fueran adecuadas o realistas, sin delegar toda la responsabilidad en algunas personas de la organización.

El centro debe tomar decisiones sobre la organización, la resolución de cuestiones administrativas y burocráticas, los horarios para la coordinación, la elección más ajustada de tener coordinador/a TIC o equipos dedicados a la tecnología en el aprendizaje y, por último, cómo se realizará el asesoramiento y la mejora general del centro educativo (Aguaded \& Tirado, 2010; Fernández, 2007; Valverde, Garrido, \& Sosa, 2010; Espuny, Gisbert, Coiduras, \& González, 2012).

La organización de los centros dependerá de las estrategias que lleven a cabo, y puede ser una estrategia de colocación de los recursos en espacios concretos o alojados en las diferentes clases. Estas estrategias organizativas determinarán la integración didáctica de las tecnologías en la dinámica natural del proceso de enseñanza y aprendizaje. Precisamente por ello, la organización, el acceso a los recursos, así como el uso a disposición del alumnado, son por sí mismos, estrategias determinantes para la metodología y su impacto en el aprendizaje (Castro, Olarte, \& Corredor, 2016). Sin embargo, aunque los centros lleven a cabo una estrategia de organización en las aulas a disposición del alumnado, la obsolescencia, la disponibilidad de los recursos, el funcionamiento o la conectividad son barreras que impiden la utilización fluida de estos dispositivos (Fernández \& Carrera, 2016; Partida, Moreno, \& Castañeda, 2015).

Es necesario tener en cuenta que al igual que el profesorado debe estar formado, las familias deben estar concienciadas de las posibilidades de las tecnologías en educación. El centro educativo debe trabajar con las familias para que com- prendan que las tecnologías son un recurso más en la práctica diaria que el alumnado dispondrá para su aprendizaje. Abrir los espacios educativos al entorno promueve la vinculación de experiencias reales del centro a las familias, con beneficios claros para el aprendizaje del alumnado (Macià \& Garreta, 2018; Arboleya \& Dopico, 2017; Llamas \& Ruiz, 2015)

Como se aprecia, las investigaciones consultadas recogen la complejidad de la integración de las tecnologías en el centro escolar. La transformación cultural, organizativa y profesional supone modificaciones en diversos niveles (Gewerc \& Montero, 2013; Cerrillo, Esteban, \& Paredes, 2014). Para ello, los centros educativos necesitan apoyo institucional por parte de los agentes externos y las instituciones administrativas, con el objetivo de que clarifiquen funciones, posibiliten estrategias distintas de organización del espacio, mejoren las condiciones laborales y profesionales, ofrezcan recursos materiales o económicos, además de disponer de formación y asesoramiento TIC en sus centros.

\section{Discusión y Conclusiones}

Comenzando con el estudio bibliométrico (análisis cuantitativo) de los dos tópicos presentados, se demuestra que hay una cantidad de producción bastante menor en el segundo tópico (coordinación, asesoramiento y la organización del centro) que en aquel destinado a la formación del profesorado. Esta diferencia puede ser debida a varios factores.

Por un lado, puede estar condicionado por la relevancia del profesorado como un agente clave para la integración coherente de las tecnologías en educación, lo cual conlleva a la investigación en la formación de éste y depositar mayor esfuerzo para analizar sus competencias TIC en vez de indagar en otros aspectos relacionados con la temática de las TIC en educación.

Por otro lado, teniendo claro que la formación del profesorado en cuestiones digitales tiene más atención que la coordinación, la comunicación, el asesoramiento o la coordinación TIC en los cen- 
tros educativos, también se debe comentar que este último tópico suele estar ligado a otras temáticas. Es decir, es menos usual encontrar recursos bibliográficos dedicados a analizar exclusivamente la relevancia del coordinador TIC en los centros escolares, las estrategias directivas u organizativas, o el papel de las familias para la innovación en tecnología. Es más común que estos aspectos de la integración de las TIC en la educación se analicen relacionados con otros aspectos como, el uso de las redes sociales, el aprendizaje colaborativo, el impacto de las TIC en el aprendizaje, entre muchas otras. Lo cual puede limitar la clasificación y el análisis concreto sobre esta temática más transversal.

Dentro del tópico de centro, encontramos que las subtemáticas más relacionadas con la coordinación y el asesoramiento en TIC, resultan ser más concretas y especializadas que la organización del espacio o las funciones del equipo directivo. A raíz de esta explicación, se debe recordar que no todas las comunidades incluyen en sus servicios agentes coordinadores TIC o asesores TIC, por lo que, por cuestiones logísticas habrá menos producción académica. Al estar menos investigado, quedan ciertas cuestiones en la ambigüedad que, puestas a debate por la comunidad educativa, bien podrían servir para crear más políticas públicas que mejoren la importante función de estos agentes claves para el proceso de transformación de los centros escolares.

Con respecto a los cambios bibliográficos que se puedan producir a lo largo de este año, entrevemos que es más posible que el tópico de "Coordinación, asesoramiento y organización TIC", llegue a superar, incluso, la cantidad obtenida en cada uno de los intervalos anteriores. En otras palabras, aunque no tenga la relevancia de la formación del profesorado, parece que indagar en el "centro escolar" tiene cada vez más atención para la investigación de las tecnologías en educación. Mientras que el tópico del centro escolar se prevé que crezca a lo largo de este año, el tópico de formación del profesorado aun estando en un nivel de producción mucho mayor, se estima que se mantendrá similar a su anterior intervalo de tiempo.

Por otro lado, con respecto a los resultados obtenidos de esta muestra concreta, podríamos afir- mar que, debido a una creciente preocupación por conocer la competencia del profesorado en el uso didáctico, técnico y pedagógico de las tecnologías, cada vez coge más fuerza las metodologías empíricas, así como la recogida de información a través de instrumentos de investigación cualitativos. Si bien es cierto que el cuestionario es un método adecuado para la recogida de información de una muestra amplia, debemos replantearnos, cuando el objetivo es conocer el nivel formativo real que tiene el profesorado, sus posibilidades y limitaciones.

El cuestionario mediría la percepción que tiene este sobre su propio nivel de uso de las tecnologías, pero eso no supone que sea realmente competente digitalmente, ya sea en los aspectos más técnicos de lo digital, así como en los aspectos didácticos y pedagógicos de la integración de las tecnologías en el sistema escolar. Es necesario cuestionar y valorar críticamente la utilización de este instrumento de recogida de información sobre las competencias, con el objetivo de reflexionar acerca de medidas alternativas de recogida de información que, o bien complementen la información, o que pongan el foco de atención en el nivel competencial real del profesorado. Sin embargo, tampoco se podría analizar la competencia digital del profesorado sin analizar el resto de sus competencias que, de forma simultánea, se desarrollan permanentemente mientras ejerce su labor educativa (Castañeda, Esteve \& Adell, 2018).

Por otro lado, el tópico de "Coordinación, asesoramiento y organización de centro TIC", se suele analizar más desde un foco humanístico-interpretativo, con la finalidad de, no solamente extraer datos y analizarlos, sino también ser capaz de realizar interpretaciones y teorías al respecto. Esta elección en la temática puede estar muy relacionada con el contexto de análisis del que se pretende extraer datos. Como hemos comentado en apartados anteriores, la investigación humanístico-interpretativa pone el foco de atención en el ambiente natural y en la descripción del contexto, lo cual puede relacionarse con que el investigador entienda que el tópico en sí mismo -por su propia naturaleza- mantiene una relación directa con un contexto concreto como el centro escolar. Es decir, si queremos conocer cómo se está llevan- 
do a cabo la coordinación TIC -por ejemplo-, la tendencia habitual es considerar como objeto de estudio el centro y se seleccionan diferentes instrumentos que recojan información de ese lugar y de las relaciones desarrolladas.

Precisamente por ello, parece que desde la investigación se entiende que la temática se presta más una relación del investigador o investigadora con el objeto de estudio desde las características idiosincráticas propias de un entorno y de los agentes que conviven en él. Por ello, los instrumentos y herramientas de recogida de la información seleccionados para analizar el segundo tópico son múltiples y más variadas, ya que encontramos un abanico mucho mayor de técnicas distintas propias de perspectivas mixtas -cuantitativas y cualitativas-.

En ambos casos, sorprende la baja presencia de las perspectivas investigadoras más dirigidas a la práctica y a la mejora. Tanto para el caso de estudio de la formación del profesorado como en la mejora de la coordinación, comunicación y organización del centro, resulta interesante esta perspectiva metodológica por el impacto directo en las posibilidades innovadoras del centro, así como que, probablemente, los intentos de cambios estarían más afinados a la realidad contextual. Según Castañeda, Esteve \& Adell (2018), la competencia digital no puede ser entendida de forma integral fuera del contexto donde se sitúa el profesorado.

Si no fuera suficiente este argumento sobre la innovación construida desde el propio seno de la realidad educativa, desde el punto de vista investigador también tiene gran interés y relevancia. El investigador o investigadora se encontraría directamente con las barreras que el profesorado, el equipo directivo o los/as coordinadores/as TIC se encuentran día a día en su centro educativo, lo que se traduce en gran cantidad de datos y experiencias a nivel de aula que en ocasiones el investigador/a no tiene o pierde por lejanía de la realidad práctica en el sistema educativo escolar. Esta alternativa de investigación orientada al cambio, podría suponer la formación del profesorado, posibilitar la involucración efectiva y real de las familias para aumentar su consciencia sobre el uso de las tecnologías en el aula, así como optimizar las funciones del equipo directivo, del coordinador/a o asesor/a TIC para la mejora integral del centro, un apoyo y aliciente para la mejora de la práctica educativa, entre muchas otras (Macià, \& Garreta, 2018; Pastor, 2016; Llamas \& Ruiz, 2015).

Aunque en este trabajo se haya separado estas dos tendencias (formación del profesorado, por un lado, y coordinación, asesoramiento y organización del centro, por otro) con el objetivo de analizarlas y clasificarlas, es conveniente recalcar la necesidad de prestar atención a ambas de forma holística (Díez-Gutiérrez \& Díaz-Nafría, 2018; Esteve, Castañeda, \& Adell, (2018). El profesorado necesita un equipo directivo que facilite cambios a niveles organizativos, le apoye en su convicción o dote de recursos para su práctica diaria, sin embargo, si el centro no modifica su comportamiento o sus prácticas normalizadas, difícilmente este profesorado innovador y formado encontrará apoyo entre sus compañeros y compañeras de centro. Por otro lado, aunque el centro deposite recursos y esfuerzos para el cambio innovador apoyado en las tecnologías como un recurso más, el profesorado debe continuar formándose junto con las personas que asumen la coordinación o el asesoramiento. Esta idea tiene sentido en la medida en la que la brecha digital primera -disposición de los recursos- ha mutado a una segunda que tiene que ver con el uso didáctico que se dan a los recursos digitales. En este sentido, disponer de tecnología no es la barrera más importante, ya que gran parte de los centros disponen de recursos gracias a las políticas implementadas o debido a los recursos que el centro compra (Sánchez-Antolín \& García, 2016; Area, Sanabria, \& Vega, 2015).

Se ha observado que el uso didáctico y las competencias del profesorado tiene un alto nivel de influencia en la superación de la segunda brecha digital (Fernández \& Carrera, 2016; Gómez, Cañas, Gutiérrez \& Martín-Díaz, 2014). Según las investigaciones consultadas, el profesorado debe atreverse a modificar su patrón conductual, incluso antes de que la tecnología esté ampliamente extendida en todas las aulas del centro (Fernández \& Fernández, 2016; Colás-Bravo \& Hernández Portero, 2014; Cortina-Pérez, Gallardo-Vigil, 
Jiménez-Jiménez, \& Trujillo-Torres, 2014). Es importante concebir que, aunque las tecnologías ofrezcan posibilidades interesantes, el profesorado debe integrarlas de forma natural en la práctica diaria, sin perder la esencia pedagógica-didáctica que debe orientar toda práctica educativa (Castañeda, Esteve, \& Adell, 2018). Mientras tanto, el equipo directivo debe estar capacitado para la búsqueda de alternativas con el objetivo de favorecer el equipamiento digital necesario.

La integración de las tecnologías supone esfuerzo, tiempo y recursos en varios sentidos. Por un lado, se necesita un periodo temporal para reorientar las funciones de coordinación del profesorado y de los agentes educativos, así como para que el profesorado reflexione sobre posibles cambios metodológicos en el uso de la tecnología. Por otro lado, se necesitan esfuerzos para fomentar una comunicación eficiente y seria en canales comunicativos adecuados, recursos analógicos y digitales que funcionen para desarrollar buenas prácticas en su aula, además de formar e involucrar también a las familias para que vivencien experiencias didácticas con tecnologías -esto reduciría resistencias por parte de las familias-. Por último, para modificar la cultura profesional y organizativa de un centro se necesita una formación ajustada a la realidad, además de apoyo institucional con el objetivo de potenciar la confianza y seguridad del profesorado. Esto ayudaría también a la inclusión de personas con algún tipo de NEAE (Tello Díaz-Maroto $\&$ Cascales, 2015). Con este fin, se necesita un equipo de profesionales capaces de mantener un nivel alto de implicación, pero también necesitan atención por parte de las Administraciones para que las condiciones laborales del profesional educativo mejoren -tanto a nivel económico como a nivel de ratios o carga burocrática-, que mejore también su formación e información sobre Materiales Didácticos Digitales (MDD) y aplicaciones metodológicas y didácticas (Moreira, Mesa, \& Navarro, 2015), además de disponer de recursos flexibles para que organicen el espacio como deseen y respondan a las necesidades del alumnado $\mathrm{y}$ de las familias.

En definitiva, las investigaciones apuntan a que los agentes educativos en general son fundamentales para el cambio educativo -profesorado, coordinadores, asesores, equipo directivo o familias- (Sánchez-Antolín \& García, 2016; Cerrillo, Esteban, \& Paredes, 2014; Fernández \& Valverde, 2014; Sobrado, Ceinos, \& Fernández, 2010; Valverde, Garrido, \& Sosa, 2010; Aguaded \& Tirado, 2010; Morales, 2008). De este modo, deben desarrollar una actitud adecuada hacia el aprendizaje y el cambio; deben capacitar a los centros de recursos analógicos, digitales y humanos para la práctica educativa; así como llevar a cabo experiencias colaborativas entre los agentes para su mejora conjunta.

\section{REFERENCIAS BIBLIOGRÁFICAS}

Almenara, J. C., \& Díaz, V. M. (2014). Posibilidades educativas de las redes sociales y el trabajo en grupo. Percepciones de los alumnos universitarios. Comunicar: Revista Científca de Comunicación y Educación, 21(42), 165-172. https://doi.org/10.3916/C42-2014-16

Álvarez-Arregui, E., Rodríguez-Martín, A.,Madrigal-Maldonado, R., Grossi-Sampedro, B.-Á., \& Arreguit, X. (2017). Ecosistemas de formación y competencia mediática: Valoración internacional sobre su implementación en la educación superior. Comunicar: Revista Científica de Comunicación y Educación, 25(51), 105-114.

https://doi.org/10.3916/C51-2017-10

Area, M., Hernández, V., \& Sosa Alonso, J.J. (2016). Modelos de integración didácticas de las TIC en el aula. Comunicar, 47, 79-87.

https://doi.org/10.3916/C47-2016-08

Area, M., \& Ribeiro, M. T. (2012). De lo sólido a lo líquido: Las nuevas alfabetizaciones ante los cambios culturales de la Web 2.0. Comunicar: Revista Científica de Comunicación y Educación, 19(38), 13-20.

https://doi.org/10.3916/C38-2012-02-01

Area, M., \& Sanabria, A. (2014). Opiniones, expectativas y valoraciones del profesorado participante en el Programa Escuela 2.0 en España. Educar, 50(1), 15-39.

https://doi.org/10.5565/rev/educar.64

Area, M., Mesa, Sanabria, A., \& Vega, A. (2015). Las políticas educativas TIC (Escuela 2.0) en las Comunidades Autónomas de España desde la visión del profesorado. Campus Virtuales, 2(1), 74-88.

Arnal, J., del Rincón, D., \& Latorre, D. (1992). Investigación Educativa. Fundamentos y metodología. Barcelona: Editorial Labor, S.A.

Ballesta, J., \& Cerezo, M.C. (2011). Familia y escuela ante la incorporación de las tecnologías de la Información y la Comunicación. Educación XX1, 14(2), 133-156. https://doi.org/10.5944/educxx1.14.2.248 
Bravo, P. C., Ramírez, T. G., \& Pons, J. de P. (2013). Juventud y redes sociales: Motivaciones y usos preferentes. Comunicar: Revista Científica de Comunicación y Educación, 20(40), 15-23. https://doi.org/10.3916/C40-2013-02-01

Castañeda, L., Esteve, F. \& Adell, J. (2018). ¿Por qué es necesario repensar la competencia docente para el mundo digital?RED. Revista de Educación a Distancia, 56. https://doi.org/10.6018/red/56/6

Castro Garcia, D., Olarte Dussán, F., \& Corredor, J. (2016). Tecnología para la comunicación y la solución de problemas en el aula: Efectos en el aprendizaje significativo. Digital Education Review, (30), 207-219.

Cerrillo, R., Esteban, R., \& Paredes, J. (2014). TIC e inclusión en aulas de educación secundaria de la comunidad de madrid: Análisis de las prácticas docentes en el modelo 1a 1. Profesorado: Revista De Curriculum Y Formación Del Profesorado, 18 (3), 81-97.

Decuypere, M. \& Simons, M. (2016). Sobre el potencial crítico de los enfoques sociomateriales en educación. Teoría de la Educación. Revista Interuniversitaria, 28(1), 25.

https://doi.org/10.14201/teoredu20162812544

Durán, M., Gutiérrez, I. \& Díez-Gutiérrez, E., \& DíazNafría, J.-M. (2018). Ecologías de aprendizaje ubicuo para la ciberciudadanía crítica. Comunicar: Revista Científica de Comunicación y Educación, 26(54), 49-58.

https://doi.org/10.3916/C54-2018-05

Esteve, F., Castañeda, L. \& Adell, J. (2018). Un modelo holístico de competencia docente para el mundo digital. Revista Interuniversitaria de Formación del Profesorado, 32(1)

Fernández Sánchez, M., \& Valverde Berrocoso, J. (2014). Comunidades de práctica: Un modelo de intervención desde el aprendizaje colaborativo en entornos virtuales. Comunicar: Revista Científica Iberoamericana De Comunicación Y Educación, (42), 97-105. https://doi.org/10.3916/C42-2014-09

Fernández-Ríos, L. \& Buela-Casal, G. (2009). Standards for the preparation and writing of Psychology review articles International Journal of Clinical and Health Psychology, 9(2), 329-344. Recuperado de http://www.redalyc.org/articulo. oa?id=33712028010

Ferrari, A. (2013). DIGCOMP: A framework for developing and understanding digital competence in Europe. Sevilla: Institute for Prospective Technological Studies (IPTS), European Commission. Recuperado de http://publications. jrc.ec.europa.eu/repository/bitstream/JRC83167/lb-na26035-enn.pdf

Ferrés, J., \& Piscitelli, A. (2012). La competencia mediática: propuesta articulada de dimensiones e indicadores. Comunicar: Revista Científica de Comunicación y Educación, 19(38), 75-82. https://doi.org/10.3916/C38-2012-02-08

Gewerc, A., \& Montero, L. (2013). Culturas, formación y desarrollo profesional. la integración de las TIC en las instituciones educativas. ; Cultures, Training and Career Development.the Integration of ICT in Educational Institutions. Revista de Educación, (362), 323-347.
https//doi.org/10.4438/1988-592X-RE-2011-362-163.

Guirao-Goris, J., Olmedo, A., \& Ferrer, E. (2008). El artículo de revisión. Revista Iberoamericana de Enfermeria Comunitaria. Recuperado de http://revista.enfermeriacomunitaria.org/articuloCompleto.php?ID=7.

Hernández Rivero, V., \& Sosa Alonso, J. (2016). El asesoramiento en el proceso de implantación de las TIC en las escuelas. funciones y estrategias de intervención de los asesores especialistas. RELATEC: Revista Latinoamericana de Tecnología Educativa, 15(1), 31-43.

Hershkovizt, A., \& Forkosh-Baruch, A. (2017). La relación profesor-alumno y la comunicación en Facebook: percepciones de los alumnos. Comunicar: Revista Científica de Comunicación y Educación, 25(53), 91-101.

https://doi.org/10.3916/C53-2017-09

INTEF. (2017). Marco Común de Competencia Digital Docente. Ministerio de Educación, Cultura y Deporte. Recuperado de https://aprende.intef.es/sites/ default/files/2018-05/2017_1020_Marco-Com\%C3\%BAn-de-Competencia-Digital-Docente.pdf

Llamas, F. \& Ruiz, F. J. (2015). La coordinación TIC y la formación del profesorado como elementos impulsores de la renovación pedagógica en el centro educativo, Enseñanza \& Teaching: Revista Interuniversitaria De Didáctica, 33(2), 105-121. https://doi.org/10.14201/et2015332105121

Macià Bordalba, M. \& Garreta Bochaca, J. (2018). Accesibilidad y alfabetización digital: barreras para la integración de las TIC en la comunicación familia/escuela. Revista de Investigación Educativa, 36(1), 239-257.

https://doi.org/10.6018/rie.36.1.290111

Martín, A. G., \& Tyner, K. (2012). Educación para los medios, alfabetización mediática y competencia digital. Comunicar: Revista Científica de Comunicación y Educación, 19(38), 31-39. https://doi.org/10.3916/C38-2012-02-03

Morales, O. L. (2008). Los equipos directivos de educación primaria ante la integración de las TICs. Pixel-Bit: Revista de Medios y Educación, (33), 91-110.

Muñoz-Repiso, A. G.-V., Gómez-Pablos, V. B., \& García, C. L. (2014). Las TIC en el aprendizaje colaborativo en el aula de Primaria y Secundaria. Comunicar: Revista Científica de Comunicación y Educación, 21(42), 65-74. https:// doi.org/10.3916/C42-2014-06

Partida, S. P., Arellano, C. I. M., \& Castañeda, R. H. (2015). Barreras para la integración de buenas prácticas con TIC. Estudio de caso. Innoeduca: international journal of technology and educational innovation, 1(2), 80-90. https://doi.org/10.20548/innoeduca.2015.v1i2.1044

Pastor, V. M. L. (2016). Veinte años de formación permanente del profesorado, investigación-acción y programación por dominios de acción motriz. Retos: nuevas tendencias en educación física, deporte y recreación, (29), 270-279. Recuperado de https://dialnet.unirioja.es/servlet/articulo?codigo $=5400882$

Pérez, H. S., Fernández, S. R., \& Braojos, C. G. (2010). Metodologías que optimizan la comunicación en entornos 
Integración de las TIC en la educación escolar: Importancia de la coordinación, la formación y la organización interna de los centros educativos desde un análisis bibliométrico

de aprendizaje virtual. Comunicar: Revista Científica de Comunicación y Educación, 17(34), 163-171. https://doi. org/10.3916/C34-2010-03-16

Pérez-Rodríguez, A., \& Ponce, Á. D. (2012). De la competencia digital y audiovisual a la competencia mediática: dimensiones e indicadores. Comunicar: Revista Científica de Comunicación y Educación, 20(39), 25-34. https://doi. org/10.3916/C39-2012-02-02

Prendes, M.P. (2016). Análisis conceptual de modelos decompetencia digital del profesorado universitario. RELATEC: Revista Latinoamericana de Tecnología Educativa, 15(1), 97-114. https://doi.org/10.17398/1695-288X.15.1.97

Ramírez-Montoya, M. S., \& García-Peñalvo, F.-J. (2018). Co-creación e innovación abierta: Revisión sistemática de literatura. Comunicar: Revista Científica de Comunicación y Educación, 26(54), 09-18. https://doi.org/10.3916/C54-2018-01

Sánchez Antolín, P., \& García, M. B. (2016). La política educativa TIC de la Comunidad de Madrid (Espańa): la perspectiva del profesorado. RELATEC: Revista Latinoamericana de Tecnología Educativa, 15(1), 45-58.

Sloep, P., \& Berlanga, A. (2011). Redes de aprendizaje, aprendizaje en red. Comunicar: Revista Científica de Comunicación y Educación, 19(37), 55-64.

https://doi.org/10.3916/C37-2011-02-05

Sobrado Fernández, L. M., Ceinos Sanz, M. C., \& Fernández Rey, E. (2010). Planificación y desarrollo de un mapa de habilidades TIC en orientación. Comunicar: Revista Científica Iberoamericana De Comunicación y Educación, (35), 167-174.

https://doi.org/10.3916/C35-2010-03-10

Tello Díaz-Maroto, I., \& Cascales Martínez, A. (2015). Las TIC y las necesidades específicas de apoyo educativo: análisis de las competencias TIC en los docentes.

https://doi.org/10.5944/ried.18.2.13536

Tur, G., Marín-Juarros, V., \& Carpenter, J. (2017). Uso de Twitter en Educación Superior en Espańa y Estados Unidos. Comunicar: Revista Científica de Comunicación y Educación, 25(51), 19-28. https://doi.org/10.3916/C51-2017-02

Tuzel, S., \& Hobbs, R. (2017). El uso de las redes sociales y la cultura popular para una mejor comprensión intercultural. Comunicar: Revista Científica de Comunicación y Educación, 25(51), 63-72.

https://doi.org/10.3916/C51-2017-06

Valverde, J., C. Garrido, M., \& José Sosa Díaz, M. (2010). Políticas educativas para la integración de las TIC en Extremadura y sus efectos sobre la innovación didáctica y el proceso enseñanza-aprendizaje: la percepción del profesorado.

Velandia-Mesa, C., Serrano-Pastor, F.-J., \& Martínez-Segura, M.-J. (2017). La investigación formativa en ambientes ubicuos y virtuales en Educación Superior. Comunicar: Revista Científica de Comunicación y Educación, 25(51), 09-18.

https://doi.org/10.3916/C51-2017-01

Waliño-Guerrero, Ma José; Pardo Baldoví, M. Isabel; Esnaola Horacek, Graciela \& San Martín Alonso, Ángel
(2018). "La participación escolar de las familias a través de plataformas digitales". @tic revista d'innovació educativa, 20, $80-88$

https://doi.org/10.7203/attic.20.12131

Méndez Garido, J. M., \& Delgado García, M. (2016). Las TIC en centros de educación primaria y secundaria de andalucía. un estudio de casos a partir de buenas prácticas; ICT in Primary and Secondary Education Schools in Andalusia. A caso study from best p. Digital Education Review, (29), 134-165. 


\section{Anexos}

1. Aguaded, J. I. y Tirado, R (2010). Ordenadores en los pupitres: Informática y telemática en el proceso de enseñanza-aprendizaje en los centros tic de andalucía. Pixel-Bit, (36), 5-28.

2. Alonso, Á. S. M., \& Chacón, J. P. (2014). Centros educativos inteligentes, luces y sombras sobre las políticas de transferencia tecnológica y las prácticas docentes. Profesorado: Revista de curriculum y formación del profesorado, 18(3), 64-79.

3. Arboleya, E. y Dopico, E. (2017). Superando las barreras físicas del aula: recursos naturales y TIC. Overcoming the physical barriers of the classroom: Natural resources and ICT. Revista Iberoamericana de Educación, (75), 71-88

4. Espuny Vidal, C., Gisbert Cervera, M., Coiduras Rodríguez, J. L., \& González Martínez, J. (2012). El coordinador TIC en los centros educativos: Funciones para la dinamización e incorporación didáctica de las tic en las actividades de aprendizaje. Pixel-Bit: Revista De Medios Y Educación, (41), 7-18. Recuperado de ttp://acdc.sav.us.es/pixelbit/images/stories/p41/01.pdf

5. Fernández Rodrigo, L. ; Carrera Farran, X. (2016). Aspectos organizativos y técnicos para la integración de tabletas digitales en centros de educación primaria y secundaria de Cataluña. EDUTEC. Revista Electrónica de Tecnología Educativa, (58)

6. Fernández Tilve, M. D. (2007). ¿Contribuyen las TIC a hacer de los profesores mejores profesionales?: ¿Qué dicen los directivos escolares gallegos? Pixel-Bit: Revista De Medios Y Educación, (30), 5-15.

7. Hernández Rivero, V. M, Castro León F. M., Vega Navarro. (2011). El coordinador TIC en la escuela: Análisis de su papel en procesos de innovación. Profesorado, 15(1), 315-327.

8. Hernández Rivero, V. M., \& Sosa Alonso, J. J. (2016). La visión del asesor especialista en tecnología sobre el proceso de implantación de las TIC en los centros educativos, su impacto en el aprendizaje y su organización institucional. Profesorado: Revista De Curriculum y Formación Del Profesorado, 20(3), 433-466.

9. Jiménez de, A. A., León Guereño, M. S., Correa Gorospe, J. M., \& Ibáńez Etxeberria, A. (2008). La gestión del cambio tecnológico en los centros educativos del país vasco: El papel de los directivos escolares. PixelBit: Revista De Medios Y Educación, (31), 39-57.

10. Lázaro Cantabrana, J. L., \& Gisbert Cervera, M. (2007). La integración de las TIC en los centros escolares de educación infantil y primaria: Condiciones previas. PixelBit: Revista De Medios Y Educación, (28), 27-34.

11. Llamas Salguero, F. y Ruiz Peńa, F. J. (2015). La coordinación TIC y la formación del profesorado como elementos impulsores de la renovación pedagógica en el centro educativo, Enseñanza \& Teaching: Revis- ta Interuniversitaria De Didáctica, 33(2), 105-121. doi:10.14201/et2015332105121.

12. Macià Bordalba, M. y Garreta Bochaca, J. (2018). Accesibilidad y alfabetización digital: barreras para la integración de las TIC en la comunicación familia/escuela. Revista de Investigación Educativa, 36(1), 239-257. DOI: http://dx.doi.org/10.6018/rie.36.1.290111

13. Marta-Lazo, Carmen; García Estebaranz, Beatriz. (2017). "Herramientas virtuales para la comunicación directa entre los agentes de la comunidad educativa. Análisis de caso de la aplicación móvil Smart Schools". en @tic. revista d'innovació educativa. Número 19. Otoño (Julio-Diciembre 2017), pp. 1-9.

14. Martí, R., Gisbert, M. \& Larraz, V. (2018). Ecosistemas tecnológicos de aprendizaje y gestión educativa. Caracteristicas estratégicas para un diseño eficiente. EDUTEC, Revista Electrónica de Tecnología Educativa, 64. Recuperado de: http://dx.doi.org/10.21556/ edutec.2018.64.1025

15. Morales Castro, O., Lorenzo Delgado, M., \& Trujillo Torres, J. M. (2010). Los equipos directivos de educación primaria ante la integración de las TICs. Pixel-Bit: Revista De Medios Y Educación, (33), 91-110.

16. Pérez, M. E. del M., Martínez, L. V., \& Piñeiro, M. del R. N. (2014). Variables asociadas de la cultura innovadora con TIC en escuelas rurales. Profesorado: Revista de curriculum y formación del profesorado, 18(3), 9-25.

17. Beneyto-Seoane, Mar; Collet-Sabé, Jordi (2016). "Las relaciones digitales entre familias y escuela: análisis y propuestas” en @tic. revista d'innovació educativa, (16), 1-9.

18. Sobrado Fernández, L. M., Ceinos Sanz, M. C., \& Fernández Rey, E. (2010). Planificación y desarrollo de un mapa de habilidades TIC en orientación. Comunicar: Revista Científica Iberoamericana De Comunicación y Educación, (35), 167-174.

19. Sosa Díaz, Ma J., Peligros García, S. y Díaz Muriel, D. (2010). Organizational good practices for the integration of the ict in the education system of extremadura buenas prácticas organizativas para la integración de las tic en el sistema educativo extremeño. Teoría De La Educación : Educación Y Cultura En La Sociedad De La Información, 11(1), 148-179.

20. Valverde-Berrocoso J., \& Sosa-Díaz, M. J. (2014). Centros educativos e-competentes en el modelo $1: 1$. el papel del equipo directivo, la coordinación tic y el clima organizativo. Profesorado, 18(3), 41-62. http:// www.ugr.es/ $\sim$ recfpro/rev183ART3.pdf

21. Waliño-Guerrero, Ma José; Pardo Baldoví, M. Isabel; Esnaola Horacek, Graciela y San Martín Alonso, Ángel (2018). "La participación escolar de las familias a través de plataformas digitales". @tic revista d'innovació educativa, 20, 80-88 
Integración de las TIC en la educación escolar: Importancia de la coordinación, la formación y la organización interna de los centros educativos desde un análisis bibliométrico

22. Abad, A., , M. J. R., , N. Z., \& Bastidas, S. (2013). Tecnologias de la información y la comunicación para maestros de nivel inicial : Herramientas informáticas en la formación docente. Enseñanza De Las Ciencias: Revista De Investigación Y Experiencias Didácticas, (2013)

23. Albalejo López, M. J. (2018). Estudio de un caso de la formación del profesorado en las tecnologías de la información y la comunicación en los centros de educación infantil y primaria, Revista DIM http://dimglobal. net/revista.htm

24. Boza, Á, \& Conde, S. (2015). Training, attitude, use and impact of web 2.0 in higher education: Scale validation / formación, actitud, uso e impacto de la web 2.0 en educación superior: Validación de una escala. Cultura Y Educación, , 1-35. doi:10.1080/11356405 .2015 .1034531

25. Cabero Almenara, J. (2014). Formación del profesorado universitario en TIC. aplicación del método delphi para la selección de los contenidos formativos. Educacion XX1; University Teacher Training in ICT.Application of Delphi Method for the Selection of Training Content, 17(1), 111-131. doi:10.5944/educxx1.17.1.10707

26. Cerveró, G. A., Rodríguez, J. M. S., Meliá, J. M. J., \& Alonso, M. N. O. (2011). Las competencias y el uso de las tecnologías de información y comunicación ( TIC) por el profesorado: Estructura dimensional. Revista Electronica De Investigación Educativa; Competencies and the use of Information and Communication Technologies (ICT) by the Teaching Staff:Dimensional Structure, 13(1), 28-42.

27. Colás-Bravo, P., \& Hernández Portero, G. (2014). Incidencia de la formación del profesorado en sus creencias sobre el valor de las TIC en la enseñanza de la música. Educatio Siglo XXI, 32(3), 51. doi:10.6018/j/210981

28. Cortina-Pérez, B., Gallardo-Vigil, M., JiménezJiménez, M. Á, \& Trujillo-Torres, J. (2014). Digital illiteracy: A challenge for 21st century teachers / el analfabetismo digital: Un reto de los docentes del siglo XXI. Cultura Y Educación, , 1-34. doi:10.1080/11356 405.2014.935108

29. Cózar Gutiérrez, R., Moya Martínez, M. V., Hernández Bravo, J. A., \& Hernández Bravo, J. R. (2015). Tecnologías emergentes para la enseńanza de las ciencias sociales.: Una experiencia con el uso de realidad aumentada en la formación inicial de maestros. Digital Education Review, (27), 138-153

30. Fartura, S., Pessoa, T., \& Barreira, C. (2014). El papel de las TIC en las prácticas de los profesores de educación primaria en portugal: Estudio exploratorio. Profesorado: Revista De Curriculum Y Formación Del Profesorado, 18(3), 119-135.

31. Fernández Cruz, F. J., \& Fernández Díaz, M. J. (2016). Los docentes de la generación $\mathrm{Z}$ y sus competencias digitales. Comunicar: Revista Científica Iberoamericana De Comunicación Y Educación, (46), 97-105.
32. Fernández de la Iglesia, J. C., Fernández Morante, M. C., \& Cebreiro López, B. (2016). Desarrollo de un cuestionario de competencias en TIC para profesores de distintos niveles educativos. Pixel-Bit: Revista De Medios Y Educación, (48), 135-148.

33. Fernández Espínola, C., Ladrón-de-Guevara, L. Almagro, B. J. y Rebollo-González, J. A. (2018). Formación del profesorado de Educación Física en TIC: Modelo TPACK. EA, Escuela Abierta, 21, 65-75. doi:10.29257/EA21.2018.05

34. Francisco Álvarez, J., \& Gisbert Cervera, M. (2015). Grado de alfabetización informacional del profesorado de secundaria en Espańa: Creencias y autopercepciones. Comunicar: Revista Científica Iberoamericana De Comunicación Y Educación, (45), 187-194.

35. García, C., Días, P., Sorte, A., Díaz Pérez, J., Rita Leal, A., \& Gandra, M. (2014). El uso de las TIC y herramientas de la web 2.0 por maestros portugueses de la educación primaria y educación especial: La importancia de las competencias personales. Profesorado, 18(1), 241-255.

36. Gewerc, A., \& Montero, L. (2013). Culturas, formación y desarrollo profesional. la integración de las TIC en las instituciones educativas. Cultures, Training and Career Development.the Integration of ICT in Educational Institutions. Revista De Educacion, (362), 323347. doi:10.4438/1988-592X-RE-2011-362-163

37. Gómez Crespo, M., Cañas Cortazar, A.,M., Gutiérrez Julián, M.,S., \& Martín - Díaz, M.,J. (2014). Ordinadors a l'aula: Estem preparats els professors?; computers in the classroom. are teachers ready?; ordenadores en el aula: ¿estamos preparados los profesores? Enseńanza De Las Ciencias: Revista De Investigación Y Experiencias Didácticas, 32(2)

38. Imbernón Muñoz, F., Silva García, P., \& Guzmán Valenzuela, C. (2011). Competencias en los procesos de enseñanza- aprendizaje virtual y semipresencial. Comunicar: Revista Científica Iberoamericana De Comunicación Y Educación, (36), 107-114.

39. Llorente Cejudo, M. C., \& Toledo Morales, P. (2016). Formación inicial del profesorado en el uso de tecnologías de la información y la comunicación (TIC) para la educación del discapacitado; initial teacher training in the use of information and communications technology (ICT) for education of the sisabled. Digital Education Review, , (30), 135-146.

40. Marqués Graells, P., \& Quesada Pallarès, C.(2013): Buenas prácticas para el uso didáctico de las aulas 2.0: Formación, modelos didácticos, ventajas e inconvenientes. Educatio Siglo XXI,

41. Pegalajar Palomino, M. C. (2017). El futuro docente ante el uso de las TIC para la educación inclusiva. Digital Education Review, (31), pp.131

42. Peirats-Chacón, José; Gabaldón-Estevan, Daniel; Marín-Suelves, Diana (2018). "Percepciones sobre materiales didácticos y la formación en competencia digital”. @tic revista d'innovació educativa, 20, 54-62. 
43. Prendes Espinosa, M. P., Castañeda Quintero, L. \& Gutiérrez, I. (2010). Competencias para el uso de TIC en futuros maestros. Comunicar: Revista Científica Iberoamericana De Comunicación Y Educación, (35), 175-182.201

44. Roig Vila, R., Mengual-Andrés, S., \& Quinto-Medrano, P. (2015). Conocimientos tecnológicos, pedagógicos y disciplinares del profesorado de primaria. Comunicar: Revista Científica Iberoamericana De Comunicación Y Educación, (45), 151-159.

45. Romero-Martín, R., Castejón-Oliva, F.-J., LópezPastor, V.-M., \& Fraile-Aranda, A. (2017). Evaluación formativa, competencias comunicativas y TIC en la formación del profesorado. Comunicar, 25(52), 73-82. https://doi.org/10.3916/C52-2017-07

46. Suárez Rodríguez, J. M., Almerich, G., Gargallo López, B., \& Aliaga, F. M. (2013). Las competencias del profesorado en tic: Estructura básica (the competencies of teachers in ict: Basic structure). Educación XX1, 16(1) doi:10.5944/educxx1.16.1.716 